\title{
Evaluation of RGD functionalization in hybrid hydrogels as 3D neural stem cell culture systems
}

\author{
Emanuele Mauri ${ }^{\mathrm{a},{ }^{*}}$, Alessandro Sacchetti ${ }^{\mathrm{a}}$, Nunzio Vicario ${ }^{\mathrm{b}}$, \\ Luca Peruzzotti-Jametti $^{\text {b }}$, Filippo Rossi ${ }^{\mathrm{a}, \mathrm{o}}$ and Stefano Pluchino ${ }^{\mathrm{b}, *, o}$
}

a Department of Chemistry, Materials and Chemical Engineering "Giulio Natta", Politecnico di Milano, via Mancinelli 7, 20131 Milan, Italy

b Department of Clinical Neurosciences - Division of Stem Cell Neurobiology, Wellcome Trust-Medical Research Council Stem Cell Institute and NIHR Biomedical Research Centre, University of Cambridge, Clifford Allbutt Building - Cambridge Biosciences Campus, Hills Road, CB2 0HA, Cambridge, UK

*Emanuele Mauri. Tel.: +39 022399 3130; fax: +39 022399 3180; e-mail: emanuele.mauri@ polimi.it

* Stefano Pluchino. Tel.: +44 1223 331163; fax: +44 1223 331174; e-mail: spp24@cam.ac.uk

${ }^{\circ}$ these authors share last authorship 


\begin{abstract}
The use of neural stem cells (NSCs) in cell therapy appears as a powerful tool for the treatment of central nervous system diseases, including traumatic brain and spinal cord injuries. However, a significant drawback is related to the limited viability after transplantation in situ. The design of three-dimensional (3D) scaffolds able to resemble the architecture and the physico-chemical features of extracellular environment would be a suitable approach to improve cell survival and preserve their cellular active phase over time. In this study, we investigated NSC adhesion and proliferation in hydrogel systems; in particular, we evaluated the effect of RGD binding domains on cell fate within the polymeric scaffold. The introduction of the tripeptide, through hydrogel chemical functionalization, improved the percentage of proliferating cells until 8 days after the seeding than an unmodified scaffold. The benefic effect of this $3 \mathrm{D}$ culture system was further evident when compared to NSC monolayer (2D) culture, resulting approximately in $40 \%$ increase of cells in active phases at 4 and 8 days and maintaining a difference of $25 \%$ until 21 days after the seeding.
\end{abstract}




\section{Introduction}

The biomaterial-based therapies represent one of the last and promising approaches to interface with native tissues in predefined ways ${ }^{1-3}$. Many researchers have focused their attention on the design of biocompatible materials able to engage in a dialogue with the biological environment: a wide range of successful systems are produced for the detection of biological compounds ${ }^{4,5}$, the delivery of drugs or active substances in response to diseases ${ }^{6-8}$ and the on-demand presentation of bioactive ligands to direct cell behavior and regenerate tissues ${ }^{9,}{ }^{10}$. Particularly, the synergy of protective and repair measures could be the solution to injuries and disorders. Among them, disruptions to the central nervous system (CNS) are one of the most devastating conditions due to the severe and permanent deficits, often resulting in the loss of neuronal cell bodies, axons and associated glia support ${ }^{11,12}$. Physical consequences are commonly visible in the traumatic brain injury (TBI) and in spinal cord injury (SCI). The limited ability of the CNS to spontaneously regenerate is related to the cell inhibition around the lesion site and to the formation of a dense scar tissue and impairs axonal regeneration and functional recovery of the spinal cord. Among the SCI treatments, the transplantation of certain stem cells into the damage areas is one of the most used regenerative approaches over the last years ${ }^{13-15}$. Especially, neural stem cells (NSCs) are under investigation with regards to their support in axonal regeneration, neuroprotection or re-setting the inflammatory response to a mode that heals the damage tissue ${ }^{16-18}$. This type of cells represents a great potential tool, not only because of their capacity to integrate into the host tissue, contributing to the replacement of damaged cells but also because of several bystander capacities, such as tissue trophic support and immune-regulation ${ }^{19}$. Therefore, they are versatile and generally available from different sources, including embryonic, fetal, adult and induced pluripotent stem cells. However, some issues do exist about the low viability, the lack of control on stem cell fate, and the reduced cell conjunction with existing neural circuity after transplantation. The crucial aspect of cell survival could be approached using a three-dimensional (3D) substrate able to host cells and create a suitable biomimetic environment where they can proliferate ${ }^{20}$. 
As reported in literature ${ }^{21,22}$, a two-dimensional (2D) monolayer cells cultured on a substrate does not adequately take into account the natural environment of cells than a 3D system, which is more reflective of in vivo cellular response. The additional dimensionality of 3D cultures mimics the influence over the spatial organization of the cell surface receptors engaged in interactions with surrounding cells and also affect the signal transduction from the outside to the inside of cells, influence gene expression and cellular behavior, motility, and migration similarly to in vivo conditions. Hydrogels are an important class of highly hydrated biomaterials commonly used in drug delivery, regenerative medicine and tissue engineering thanks to their tunable physicochemical properties and their 3D structure ${ }^{23,24}$. Complying with the biocompatibility and biodegradability criteria, hydrogels can be produced through smart combinations of natural and synthetic polymers: the first ones impart biological activity and typically promote cell interaction; the second ones offer the opportunity to tailor the scaffold architecture due to their reproducible characteristics in terms of molecular weight, degradation and mechanical properties. The swelling behavior allows for the exchange of ions and metabolites with tissue fluids to maintain the biological chemical balance with the surrounding environment and the diffusion of biomolecules preserving hydrogel physical structure ${ }^{25,26}$. The porous morphology also consents the outline of the in vivo distribution of metabolites, nutrients, oxygen, and signaling molecules. Nowadays, their use as permissive microenvironments to enhance cell survival and control stem cell fate is a significant challenge, in particular in stem cell based treatments for CNS regeneration ${ }^{13,27}$. Syntheses of hydrogels with inert surface that prevent non-specific adsorption of ligands/proteins or with biological molecules covalently incorporated within the scaffolds represent two different alternatives to pursue this objective $e^{28-30}$. In this work, we investigated both these strategies: we proposed the use of two hydrogels composed by polyethylene glycol (PEG), agarose and poly acrylic acid (PAA) as a 3D culture system to preserve NSC viability over time. 
The first one was a scaffold without polymer conjugation to biomolecules, whereas the second one was characterized by the PAA functionalization with cell-adhesive and cell-recognition tripeptide Arg-Gly-Asp (RGD) through Copper-Catalyzed Azide-Alkyne Cycloaddition (CuAAC) reaction. In this case, we introduced a stable and uncleavable bond between the polymer and the RGD molecule, in order to preserve the potential effect of the functionalization in the bioenvironment over time. We monitored the cell behavior and spatial organization within the two scaffolds and compared the obtained results against a 2D NSC culture system. In particular, the NSCs trend towards the quiescent state was recorded and correlated to the cytocompatibility of the biomaterial. The design of a smart injectable system, like a hydrogel, properly functionalized, conformable to various structures and highly porous may provide the suitable conditions to simulate the in vivo cellular environment, upgrading the stem cell curative actions in situ. 


\section{Experimental}

\section{Materials}

The used polymers in hydrogel synthesis were: PAA (average $\mathrm{Mn}=130 \mathrm{kDa}, 35 \% \mathrm{w} / \mathrm{w}$ solution in water, Sigma Aldrich Chemie GmbH, Germany), carbomer 974P (MW = 1 MDa, Fagron, The Netherlands), polyethylene glycol (PEG, MW = 2 kDa, Sigma-Aldrich Chemie GmbH, Germany) and agarose $(\mathrm{MW}=300 \mathrm{kDa}$, Invitrogen, ThermoFisher Scientific, USA). Propidium iodide solution was obtained from Invitrogen, ThermoFisher Scientific (USA). Primary antibody rabbit anti-ki67 (Rb ki67) and unconjugated secondary antibody goat anti-rabbit Alexa Fluor 647 were purchased from Abcam (UK). Normal goat serum (NGS) was obtained from Sigma-Aldrich. DMEM/F12 was obtained by Life Technologies (USA). The other chemicals were purchased from Sigma-Aldrich (Germany). The materials were used as received. Solvents were of analytical grade. RGD derivative was stored at $-20^{\circ} \mathrm{C}$. The NMR experiments were carried out on a Bruker AC (400 $\mathrm{MHz})$ spectrometer using chloroform $\left(\mathrm{CDCl}_{3}\right)$ or deuterium oxide $\left(\mathrm{D}_{2} \mathrm{O}\right)$ as solvent, and chemical shifts were reported as $\delta$ values in parts per million with respect to TMS as internal standard. FT-IR analysis were recorded using a Thermo Nexus 6700 spectrometer coupled to a Thermo Nicolet Continuum microscope equipped with a 15× Reflachromat Cassegrain objective at a resolution of 4 $\mathrm{cm}^{-1}$ using the $\mathrm{KBr}$ pellet technique. About the evaluation of immunofluorescence of NPCs loaded within hydrogel systems, the frozen hydrogel slides with stained NPCs were collected onto SuperfrostPlus slides by Thermo Fisher Scientific (USA).

\section{PAA-RGD functionalization}

The chemical modification of PAA with RGD moiety occurred as discussed in our previous work ${ }^{31}$. Briefly, the procedure involved two steps. The first one was PAA functionalization with alkyne groups. 
PAA (500 mg, $6.94 \mathrm{mmol})$ was dissolved in distilled water $(15 \mathrm{ml})$ and the following reagents were sequentially added: propargylamine hydrochloride $(63.5 \mathrm{mg}, 0.69 \mathrm{mmol})$, a solution acetonitrile:water 1:1 v/v of 1-hydroxybenzotriazole hydrate $(93.2 \mathrm{mg}, 0.69 \mathrm{mmol})$ and ethyldimethylaminopropyl carbodiimide hydrochloride $(132.3 \mathrm{mg}, 0.69 \mathrm{mmol})$. After $24 \mathrm{~h}$ stirring continuously, the mixture was dialyzed against water solution at $\mathrm{pH}=5.5(2000 \mathrm{ml})$ prepared dissolving sodium chloride (11.2 g) in distilled water and adding $\mathrm{HCl} 37 \%$ (4 drops). Dialysis occurred for three days, with daily water changes and addition of $\mathrm{HCl}$. After dialysis, the obtained product (propargyl PAA) was frozen at $-20^{\circ} \mathrm{C}$ and lyophilized.

The second step was related to the $\mathrm{CuAAC}$ reaction between propargyl PAA and RGD modified azide. The peptide was synthesized manually using the stepwise solid phase Fmoc method and then linked to $\mathrm{N}_{3}$ group. The functionalized polymer $(78 \mathrm{mg}, 0.072 \mathrm{mmol})$ was dissolved in distilled water and RGD azide derivative $(25 \mathrm{mg}, 0.072 \mathrm{mmol})$ was added; the system was left under stirring at $60^{\circ} \mathrm{C}$ for $24 \mathrm{~h}$. Subsequently, it was cooled at $25^{\circ} \mathrm{C}$ and dialyzed against acid water solution (as reported in the previous step) for three days, with daily water changes, and lyophilized. The obtained product was PAA grafting RGD tripeptide through triazole bond (PAA-RGD).

\section{Hydrogel synthesis}

The synthesis of hydrogels without any chemical functionalization (hereinafter referred to as $\mathrm{HG}$ ) was performed through microwave-assisted strategy. Branched polyacrylic acid Carbomer 974P (35 mg) was dissolved in a phosphate buffered saline solution (PBS, $5 \mathrm{ml}$ ) and then added PEG (300 $\mathrm{mg}$ ) and glycerol $(50 \mu \mathrm{l})$. The mixture was stirred for $30 \mathrm{~min}$ and left to settle for $1 \mathrm{~h}$. The $\mathrm{pH}$ was adjusted to 7.4 using $\mathrm{NaOH} 1 \mathrm{M}$. The resulting solution was used in 3D network synthesis as follows: agarose $(25 \mathrm{mg})$ was added to the mixture $(5 \mathrm{ml})$ and the system subjected to electromagnetic stimulation (500 W irradiated power) for $30 \mathrm{~s}$, heating up to $80^{\circ} \mathrm{C}$, to induce condensation reactions between the carboxyl and hydroxyl groups. 
The mixing reactor was kept closed to avoid any eventual loss of solvent vapors and finally the gelling solution was poured in cylinders $(0.25 \mathrm{ml}$ of mixture in each cylinder with diameter $1.1 \mathrm{~cm})$ during cooling, until gelation.

\section{Hydrogel-RGD synthesis}

Hydrogels with RGD functionalization (labelled as HG-RGD) were prepared starting with the dissolution of Carbomer 974P (25 mg) and PAA-RGD (10 mg) in PBS solution (5 ml). Then, PEG (300 mg) and glycerol $(50 \mu \mathrm{l})$ were added and the system stirred for $30 \mathrm{~min}$. The resulting solution was left to settle for $1 \mathrm{~h}$. Hydrogels were formed through microwave-assisted reaction in the same way of HG (previous section).

\section{Swelling behavior}

To assess the swelling behavior, hydrogel samples were first immersed in PBS for about $24 \mathrm{~h}$, then freeze-dried, weighted $\left(\mathrm{W}_{\mathrm{d}}\right)$ and poured in excess of the PBS to achieve complete swelling at $37{ }^{\circ} \mathrm{C}$ in a $5 \% \mathrm{CO}_{2}$ atmosphere. The swelling kinetics were measured gravimetrically: samples were removed from the PBS at regular time points. Then, hydrogel surfaces were wiped with moistened filter paper in order to remove the excess of the solution and then weighed $\left(\mathrm{W}_{\mathrm{t}}\right)$. The swelling ratio $\left(\mathrm{Q}_{\mathrm{m}}\right)$ was calculated with the following equation:

$\mathrm{Q}_{\mathrm{m}}=\frac{\mathrm{W}_{\mathrm{t}}-\mathrm{W}_{\mathrm{d}}}{\mathrm{W}_{\mathrm{d}}} \cdot 100$

where $\mathrm{W}_{\mathrm{t}}$ is the weight of the wet hydrogel as a function of time, and $\mathrm{W}_{\mathrm{d}}$ is the weight of the dry one. 


\section{Rheology}

$\mathrm{HG}$ and HG-RGD rheological analyses were performed at $37^{\circ} \mathrm{C}$ using a Rheometric Scientific ARES instrumentation (TA Instruments, New Castle, DE, US) equipped with $30 \mathrm{~mm}$ parallel plates, with a $4 \mathrm{~mm}$ gap between them. The oscillatory responses $\left(\mathrm{G}^{\prime}\right.$, elastic modulus, and $\mathrm{G}^{\prime \prime}$, loss/viscous modulus) were determined at low values of strain (0.02\%) over the frequency range $0.1-100 \mathrm{rad} / \mathrm{s}$ 32. Dynamic Strain Sweep (DSS) tests were also performed at frequency of $20 \mathrm{rad} \mathrm{s}^{-1}$ over the strain range of $0.01-100 \%$. Pseudoplastic behavior was also investigated. The behaviors of shear stress and viscosity as a function of shear rate were examined and the linearity of the viscoelastic properties was verified.

\section{Neural Stem Cells}

NSC lines were prepared from the subventricular zone (SVZ) of 4- to 8-week-old C57/B16 female mice, as reported in previous works ${ }^{33,34}$. Briefly, mice were humanely culled by cervical dislocation followed by decapitation, the parietal bones were cut cranially to caudally using micro-surgery scissors, and the brains removed. A brain slice matrix was used to obtain $3 \mathrm{~mm}$ thick brain coronal sections starting from $2 \mathrm{~mm}$ after the anterior pole of the brain. The SVZ of the lateral ventricles was isolated from coronal sections using iridectomy scissors. Tissues derived from at least 2 mice were pooled to generate cultures. Dissected tissues were transferred to a $15 \mathrm{ml}$ tube with digestion medium [balance salt solution (EBSS, Gibco), papain (1 mg/ml, Worthington), ethylenediaminetetraacetic acid (EDTA) $(0.2 \mathrm{mg} / \mathrm{ml}$, Sigma-Aldrich) and L-cysteine $(0.2 \mathrm{mg} / \mathrm{ml}$, Sigma-Aldrich)] and incubated for $45 \mathrm{~min}$ at $37^{\circ} \mathrm{C}$ on a rocking platform. At the end of the incubation, the tube was centrifuged at $200 \mathrm{~g}$ for $12 \mathrm{~min}$, the supernatant was removed and the pellet was mechanically disaggregated with $2 \mathrm{ml}$ of EBSS. The pellet was centrifuged again at 200 $\mathrm{g}$ for $12 \mathrm{~min}$ and then dissociated with a $200 \mu \mathrm{l}$ pipette and seeded in complete growth medium (CGM). 
CGM was constituted of mouse NeuroCult ${ }^{\mathrm{TM}}$ basal medium (Stem Cell Technologies) plus mouse NeuroCult ${ }^{\mathrm{TM}}$ proliferation supplements (Stem Cell Technologies) added with $2 \mu \mathrm{g} / \mathrm{ml}$ heparin, 20 $\mathrm{ng} / \mathrm{ml}$ EGF and $10 \mathrm{ng} / \mathrm{ml} \mathrm{bFGF}$. After approximately 4-7 days, a small percentage of the isolated cells begun to proliferate, giving rise to neurospheres. When neurospheres reached the necessary dimension (150-200 $\mu \mathrm{m}$ diameter), the cells were harvested in a $15 \mathrm{ml}$ tube and centrifuged at $100 \mathrm{~g}$ for $8 \mathrm{~min}$. The supernatant was then removed and the pellet dissociated by enzymatic digestion with Accumax ${ }^{\mathrm{TM}}$ at $37^{\circ} \mathrm{C}$ for $10 \mathrm{~min}$. The number of viable cells was determined by trypan blue exclusion and viable cells were re-seeded at clonal density 8,000 cells $/ \mathrm{cm}^{2}$. NSCs were transduced in vitro using a third-generation lentiviral carrier (pRRLsinPPT-hCMV) coding for the enhanced farnesylated (f)GFP, which targets the fluorescent protein to the inner plasma membrane of transduced cells ${ }^{35}$. The functional stability of these cells (in the absence or in the presence of the lentiviral transcript) has been confirmed with clonal and population studies ${ }^{33}$. Briefly, neurospheres were harvested, dissociated to a single cell suspension and seeded at high density $\left[1.5 \cdot 10^{6}\right.$ in a T75 $\mathrm{cm}^{2}$ flask (Sigma-Aldrich)] in $5 \mathrm{ml}$ fresh medium. After $12 \mathrm{~h}, 3 \cdot 10^{6} \mathrm{~T} . \mathrm{U} . / \mathrm{ml}$ of lentiviral vectors were added and $6 \mathrm{~h}$ later additional $5 \mathrm{ml}$ of fresh medium were added. $72 \mathrm{~h}$ after viral transduction, cells were harvested and re-seeded at normal concentration. Transgene expression was measured by FACS analysis before transplantation and $>98 \%$ of cells were found to be labelled. Mycoplasma negative NSCs at passage $n \leq 25$ were used in all experiments.

\section{Cell loading}

NSCs were added directly onto HG and HG-RGD sponge-like hydrogels ${ }^{28}$. They were obtained as lyophilized scaffolds, as follows: after their synthesis, hydrogels were separately submerged in PBS solution for $2 \mathrm{~h}$, then they were frozen on dry ice for $5 \mathrm{~min}$, transferred at $-80{ }^{\circ} \mathrm{C}$ overnight and then dried in the following conditions: 0.5 mbar and $-40^{\circ} \mathrm{C}$. 
The resulting lyophilized gels were sterilized for at least $30 \mathrm{~min}$, each side, by UV illumination before cells seeding. NSCs were harvested and suspended at a density of $1.25 \cdot 10^{6}$ cells $/ \mathrm{ml}$ in growth medium. An aliquot of $40 \mu \mathrm{l}$ of cells were added directly onto one side of the freeze-dried hydrogel sample and placed in incubator at $37^{\circ} \mathrm{C}$ for $20 \mathrm{~min}$; subsequently, other $40 \mu \mathrm{l}$ were added on the other gel side. Hydrogels were let to swell for $30 \mathrm{~min}$ in incubator at $37{ }^{\circ} \mathrm{C}$; then, they were placed in a 24 multiwell cell culture plate and $1 \mathrm{ml}$ of growth medium was added. The loaded samples were stored and monitored in incubator at $37^{\circ} \mathrm{C}$, replacing medium every two days. Each hydrogel contained approximately 100,000 cells. Cell seeding in 2D substrate was performed as follows: after placing one round glass coverslips in each well of a 24 multiwell plate, laminin was added to cell suspension in growth medium (1:100) and the resulting cell solution was poured to each glass $(80 \mu \mathrm{l})$ and incubate for $30 \mathrm{~min}$ at $37^{\circ} \mathrm{C}$. In this way, we replicated the same amount of cells within hydrogel samples in 2D experiments. Then, a solution of laminin and growth medium (1:100) was added $(500 \mu \mathrm{l})$ to each well and the resulting systems were stored and monitored in incubator at $37^{\circ} \mathrm{C}$, replacing medium every two days.

\section{Cellular immunofluorescence in vitro}

Immunofluorescence on NSCs in vitro was performed as described. Hydrogel medium was replaced with a solution of propidium iodide (PI) $500 \mathrm{nM}(1 \mathrm{ml})$ and the system incubated at $37^{\circ} \mathrm{C}$ for $5 \mathrm{~min}$. Then, the samples were rinsed with PBS $1 \mathrm{X}(1 \mathrm{ml})$ five times in order to remove any free dye remaining in the solution. The gels were stored in incubator during each washing, for 8 min. Finally, they were incubated for other $5 \mathrm{~min}$ at $37^{\circ} \mathrm{C}$. PBS solution was removed and cells were fixed using paraformaldehyde (PFA) solution $4 \%$ added to each hydrogel well for 10 min. Each sample was rinsed three times with PBS $1 \mathrm{X}$, and then incubated for $2 \mathrm{~h}$ at room temperature (r.t.) using a blocking solution (300 ml, composed by PBS $1 \mathrm{X}, 0.1 \%$ Triton X-100 and $10 \%$ normal goat serum) to avoid a-specific binding of the used antibodies in intracellular staining. 
The resulting HG and HG-RGD with fixed NSCs were treated with an appropriate primary antibody diluted in PBS 1X (300 ml), overnight at $4^{\circ} \mathrm{C}$. The solution was composed by PBS $1 \mathrm{X}$, with $0.1 \%$ Triton X-100, 1\% NGS and antibody Rb ki67 (1:500). The samples were then washed two times using a solution $0.1 \%$ Triton $\mathrm{X}-100$ in PBS $1 \mathrm{X}$ and incubated for $1 \mathrm{~h}$ at r.t. with the secondary antibody solution $(300 \mu \mathrm{l})$ prepared with PBS 1X, 0.1\% Triton X-100, 1\% NGS and anti-rabbit Alexa Fluor 647 (1:1000). Each hydrogel was washed three times with $0.1 \%$ Triton X-100 in PBS $1 \mathrm{X}$ solution and the NPCs nuclei were stained with 4,6-diamine-2-fenilindole ( $1 \mu \mathrm{l} / \mathrm{ml}$, DAPI) for 3 min. Finally, the samples were washed in PBS $1 \mathrm{X}$ three times. The obtained HG and HG-RGD systems were embedded in Optimal Cutting Temperature (OCT) Tissue Tek compound and snap frozen in dry ice. Frozen blocks were placed in a cryostat (Leica) and $30-\mu \mathrm{m}$ thick sections were cut and collected onto SuperfrostPlus microscope slides. The latter were then mounted with Fluorescent mounting medium. Samples were examined and photographed using a laser scanning confocal microscope TCS-SP2 (Leica Inc., Wetzlar). In order to analyze cell distribution throughout the whole gel, six sections of each $\mathrm{HG}$ and HG-RGD spaced $60 \mu \mathrm{m}$ were acquired at $63 \mathrm{x}$ magnification. The immunofluorescence and viability analyses were performed at 1, 4, 8, 14 and 21 days after NSC seeding on sponge-like gel samples. The immunofluorescence of laminin samples was performed in the same manner of hydrogels, but after the last washing in PBS 1X solution, the round glass coverslips with cells and laminin were directly mounted with Fluorescent mounting medium and evaluated at the confocal microscope.

\section{LDH assay}

The determination of released lactate dehydrogenase (LDH) was performed using the medium of hydrogels HG, HG-RGD and laminin (conditioned medium) after the cell seeding, at defined time points $(1,4,8,14,21$ days). The collected medium was used as supernatant for new NPCs cultures. 
After incubation, the resulting systems were collected, then centrifuged at $600 \mathrm{~g}$ for $10 \mathrm{~min}$. Poured the supernatant in a 96-well plate (20 $\mu \mathrm{l}$ each well), $\mathrm{LDH}$ release reagent (30 $\mu \mathrm{l})$ was added to mix completely, then incubated at r.t. for $30 \mathrm{~min}$. Cell cytotoxicity was measured by the absorbance $(490 \mathrm{~nm})$ and the results were compared with that of the control wells to determine relative cell viability.

\section{Statistical analyses}

Statistical analyses were performed by Prism software (Graph-Pad). Applied statistical tests were two-way ANOVA followed by Tukey's post hoc test, as reported in figure legends. Data are presented as mean $\pm \mathrm{SEM}$. 


\section{Results and Discussion}

\section{Hydrogel chemical characterization}

The difference between HG and HG-RGD samples is related to the use of PAA-RGD component in their synthesis. RGD is the most effective and commonly employed peptide sequence for stimulated cell adhesion on synthetic substrates due to its ability to mimic cell adhesion proteins, bind to cellsurface receptors and its biological impact on cell behavior and survival ${ }^{36-38}$. The design of a stable RGD motif linker to the synthetic surface is the focal point to achieve strong cell adhesion: many researchers proposed a covalent attachment to the polymer, exploiting functional groups like hydroxyl, amino or carboxyl groups, already available in surface polymer structure or specifically introduced by blending, copolymerization, chemical or physical treatments. In order to avoid any potential bond liability between RGD and PAA, we proposed a click chemistry approach: PAA modification with alkyne moieties and the grafting of RGD to an azide group ${ }^{31}$ allowed the formation of triazole through the $\mathrm{CuAAC}$ cycloaddition. Triazole is stable in metabolic and chemical degradations $^{39,40}$ and uncleavable under physiological conditions. FT-IR spectrum (ESI, Figure S1) shows the characteristic peaks of triazole ${ }^{41}$ at $1445 \mathrm{~cm}^{-1}$, whereas the wavenumbers range $3600-2900 \mathrm{~cm}^{-1}$ corresponding to the stretching vibration of $-\mathrm{OH}$ residual groups, with a partial overlap with the peak of $\mathrm{C}-\mathrm{H}$ stretching at $2950-2850 \mathrm{~cm}^{-1}$. The carbonyl stretch $\mathrm{C}=\mathrm{O}$ is detectable around $1700 \mathrm{~cm}^{-1}$, PAA $-\mathrm{CH}_{2}$ stretching is visible in the range $1435-1300 \mathrm{~cm}^{-1}$ and the signal at $1256 \mathrm{~cm}^{-1}$ is representative of C-O vibration. As reported in our previous work ${ }^{31}$, the functionalization of PAA occurred modifying only $10 \%$ of its carboxyl groups with triple bond, in order to preserve the residual groups for hydrogel synthesis: in this way, PAA-RGD took part to the formation of the 3D HG-RGD polymeric scaffold and it was not physically entrapped within the mesh. This approach allowed to design an alternative scaffold to $\mathrm{HG}$, attributing the potential variations in NPCs behavior to the grafted RGD. 
HG e HG-RGD hydrogels were produced through chemical cross-linking between carbomer (and PAA-RGD in HG-RGD samples), PEG and agarose, by microwave-assisted polycondensation. This chemistry is based on the efficient heating of materials by "microwave dielectric heating" effects and is dependent on the ability of a specific component (solvent or reagent) to absorb microwave energy and convert it into heat ${ }^{32,42}$. Heating to $80{ }^{\circ} \mathrm{C}$ leads to a higher macromer mobility, and thus enhances short-range interconnections among functional groups of the polymers: in details, carboxyl groups and hydroxyl groups produce local networks which, as the esterification proceeds, give rise to the final three-dimensional macrostructure. The gel forming is progressive, reaching before the sol/gel transition temperature and then the complete gelation (within $10 \mathrm{~min}$ ), avoiding damages to the structures of the components. This peculiar feature allows such hydrogels to complete their gelation directly in situ, being injectable still at the sol state, thanks to their thixotropic nature ${ }^{31,32}$. The used polymers are well known as biocompatible materials for therapeutic and tissue engineering approaches, thanks to their anti-inflammatory and clinical properties and promising interaction with cells ${ }^{1,43-45}$. The building blocks of macromolecules form a stable structure made up mostly of C-C bonds, with ester bonds as cross-linking points and, in HGRGD sample, the presence of the grafted tripeptide. A schematic representation of the two synthetized hydrogels is reported in Figure 1A. 
A

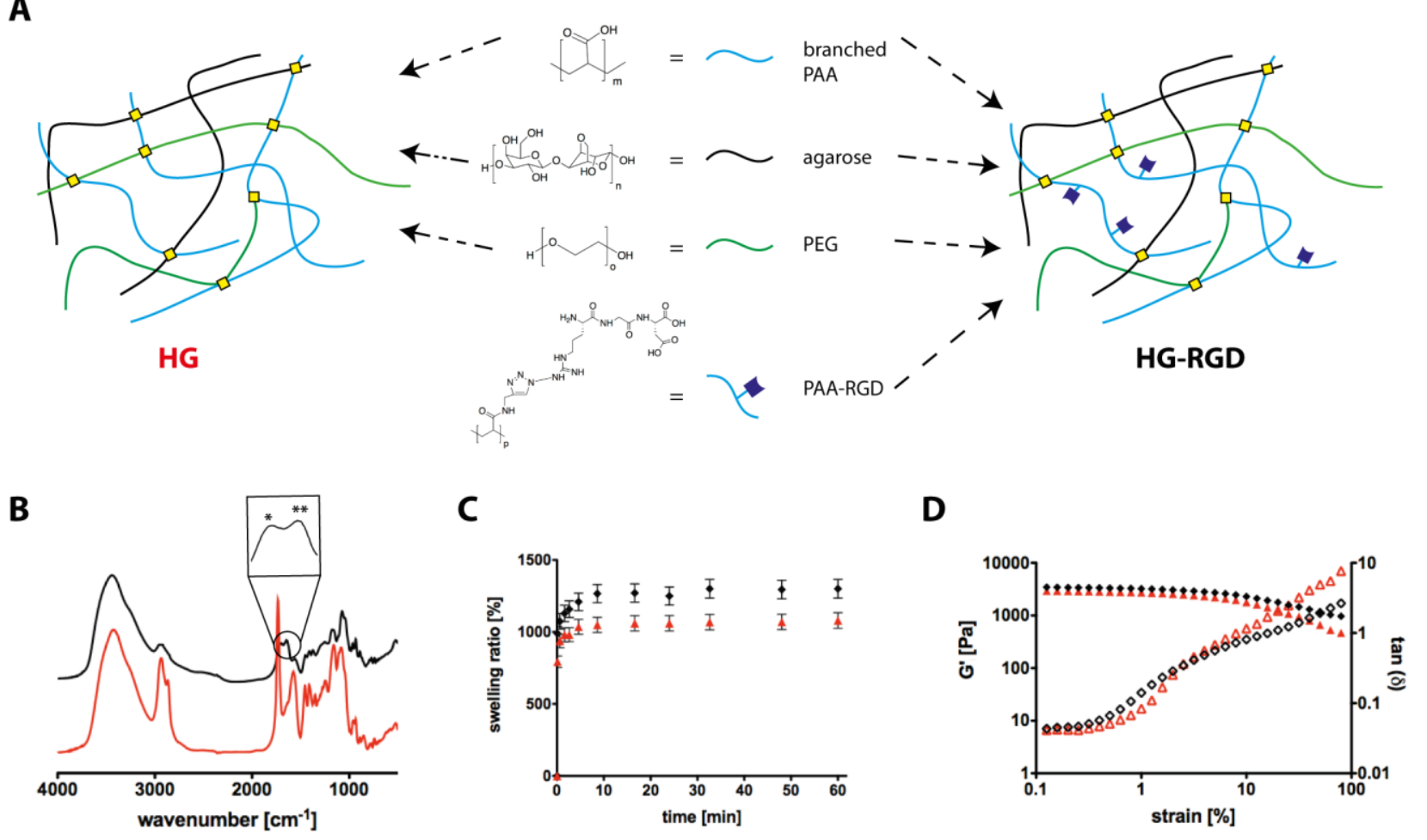

Figure 1. Hydrogels physico-chemical characterization. A: Schematic representation of unmodified (HG) and RGD-functionalized (HG-RGD) hydrogels. B: FT-IR spectra of HG (red) and HG-RGD (black); RGD amide I $(*)$ and II $(* *)$ signals are emphasized in HG-RGD spectrum. C: Swelling kinetics of HG ( $\boldsymbol{\Delta}$, red) and HG-RGD ( $\boldsymbol{\Lambda}$, black): the equilibrium was reached in the first $30 \mathrm{~min}$ and maintained over time. D: Rheological behavior of HG $\left(\mathrm{G}^{\prime} \boldsymbol{\Delta}\right.$ red, $\tan (\delta) \Delta$ red $)$ and HG-RGD $\left(\mathrm{G}^{\prime} \diamond\right.$ black, $\tan (\delta) \diamond$ black $)$ hydrogels.

The formation of ester bonds in HG and HG-RGD is visible in their FT-IR spectra (Figure 1B), where peaks corresponding to symmetric (around $1600 \mathrm{~cm}^{-1}$ ) and asymmetric (around $1400 \mathrm{~cm}^{-1}$ ) $\mathrm{CO}_{2}$ stretches are detectable. The two spectra also show the signals related to C-O-C stretch vibration (range 900-1000 $\mathrm{cm}^{-1}$ ) that represents the glycosidic bond between monosaccharide units (typical of agarose structure) and ester groups. In HG-RGD sample, the presence of RGD functionalization is confirmed by its detectable peaks corresponding to amide $\mathrm{I}^{*}{ }^{*}, 1670 \mathrm{~cm}^{-1}, \mathrm{C}=\mathrm{O}$ stretching) and amide II ( ${ }^{* *}, 1560 \mathrm{~cm}^{-1}, \mathrm{~N}-\mathrm{H}$ bending) bands ${ }^{46}$, in addition to triazole signal. 
These considerations show that the synthesis of the gel scaffold was correctly achieved and the discussed tripeptide functionalization was successfully obtained.

\section{Hydrogel physical characterization}

Hydrogels are polymeric scaffolds able to link and retain high amount of water, giving them the advantage over other matrices of better mimicking the aqueous environment of the extracellular matrix $(\mathrm{ECM})^{47}$. During swelling of a hydrogel network, polymer chains assume a stretched conformation, counterbalanced by an elastic force acting in the opposite direction generated by increasing the elongation of the system; this force limits the stretching process. On the other hand, polymer swelling is promoted by the polymer-solvent mixing process, which lowers the total free energy by increasing system entropy. The action of these opposite forces leads to the attainment of a thermodynamic equilibrium. Experimental results about HG and HG-RGD samples show that all samples exhibited fast swelling kinetics and their swelling equilibrium was reached within $30 \mathrm{~min}$. In particular, Figure 1C highlights similar swelling trend for HG (in red) and HG-RGD (in black): the first one reached a swelling ratio equal to $1100 \%$ and the second one was characterized by a value of about $1350 \%$. As reported in previous works ${ }^{23,31,48}$, swelling phenomenon exhibits high dependence on the solute loaded within the polymeric network. In this case this dependence is present, but less visible, because RGD peptide is integral with polymeric network and does not create high hindrance to gelation process. This suggested that the PAA chemical modification did not significantly affect the physical property of the network, despite in HG-RGD the functionalization decreases the number of reactive sites with a consequent decrease in cross-linking density. Furthermore, as previously demonstrated ${ }^{49}$, gels swelling kinetics is much faster than the degradation one. About rheology (Figure 1D), for both samples storage modulus $\left(\mathrm{G}^{\prime}\right)$ was found to be approximately one order of magnitude higher than the loss modulus $\left(\mathrm{G}^{\prime \prime}\right)$, indicating an elastic rather than viscous material. Both moduli were also essentially independent of frequency. 
In detail, $\mathrm{G}^{\prime}$ for $\mathrm{HG}$ and $\mathrm{HG}-\mathrm{RGD}$ were respectively $3000 \mathrm{~Pa}$ and $3500 \mathrm{~Pa}$. The RGD modified material presented stiffer and more elastic properties than $\mathrm{HG}$, that could be attributed to the interactions between polymeric network and RGD pendant groups. Also in this case the influence of RGD does not irreversibly change the rheological properties due to the integration of polymer chains and absence of high steric hindrance. The DSS test showed that both HG and HG-RGD behaviors were dominated at low strain values by the elastic modulus. Increasing the strain values, the elastic structure of the network breaks down and the elastic modulus decreases steeply. The crossover strain $\left(\gamma_{c}\right)$ can be evaluated as the value at which the contribution of the material damping $\tan (\delta)$ is predominant with respect to $\mathrm{G}^{\prime}$. At low values of strain, the two $\mathrm{G}^{\prime}$ trends indicate the presence of a close packed polymeric network and the behaviors of $\tan (\delta)$ are very similar, thus indicating very similar, liquid-like behaviors. Our previous works also revealed that dried hydrogels possess a highly entangled structure ${ }^{31}$. There are some bigger pores containing small pores and some fibrillar networks on the pore walls. In addition, most of the pores are interconnected, giving rise to a porous structure with a complex 3D construction.

\section{Hydrogel effect on NSC viability in vitro}

We tested the impact of a 3D structure on NSCs, compared to a NSC monolayer system (2D) on laminin. We also investigated how the presence of RGD motifs could improve cell adhesion and viability. As reported in literature $21,50,51,3 \mathrm{D}$ cell culture systems represent more accurately the microenvironment where cells reside in tissues and provide more predictive data for in vivo experiments. The same amounts of NSCs were seeded over the 2D substrate and within hydrogels and monitored after 1, 4, 8, 14 and 21 days in order to study cellular behavior over time. Propidium iodide was used to detect dead cells and its staining protocol was applied before NSC fixation with PFA, whereas ki67 was chosen as marker for cells in non-quiescent state, including those in proliferating active phase. It was conjugated to a secondary antibody with different 
absorption/emission spectra than PI, GFP membrane and DAPI nuclear staining, avoiding overlaps in recorded confocal images.

Figure 2 shows NSC spatial organization and proliferation on laminin (Figures 2Aa, Bb, Cc, Dd, Ee) and within HG (Figures 2Ff, Gg, Hh, Ii, Jj) and HG-RGD (Figures 2Kk, Ll, Mm, Nn, Oo) networks, at the indicated time points.

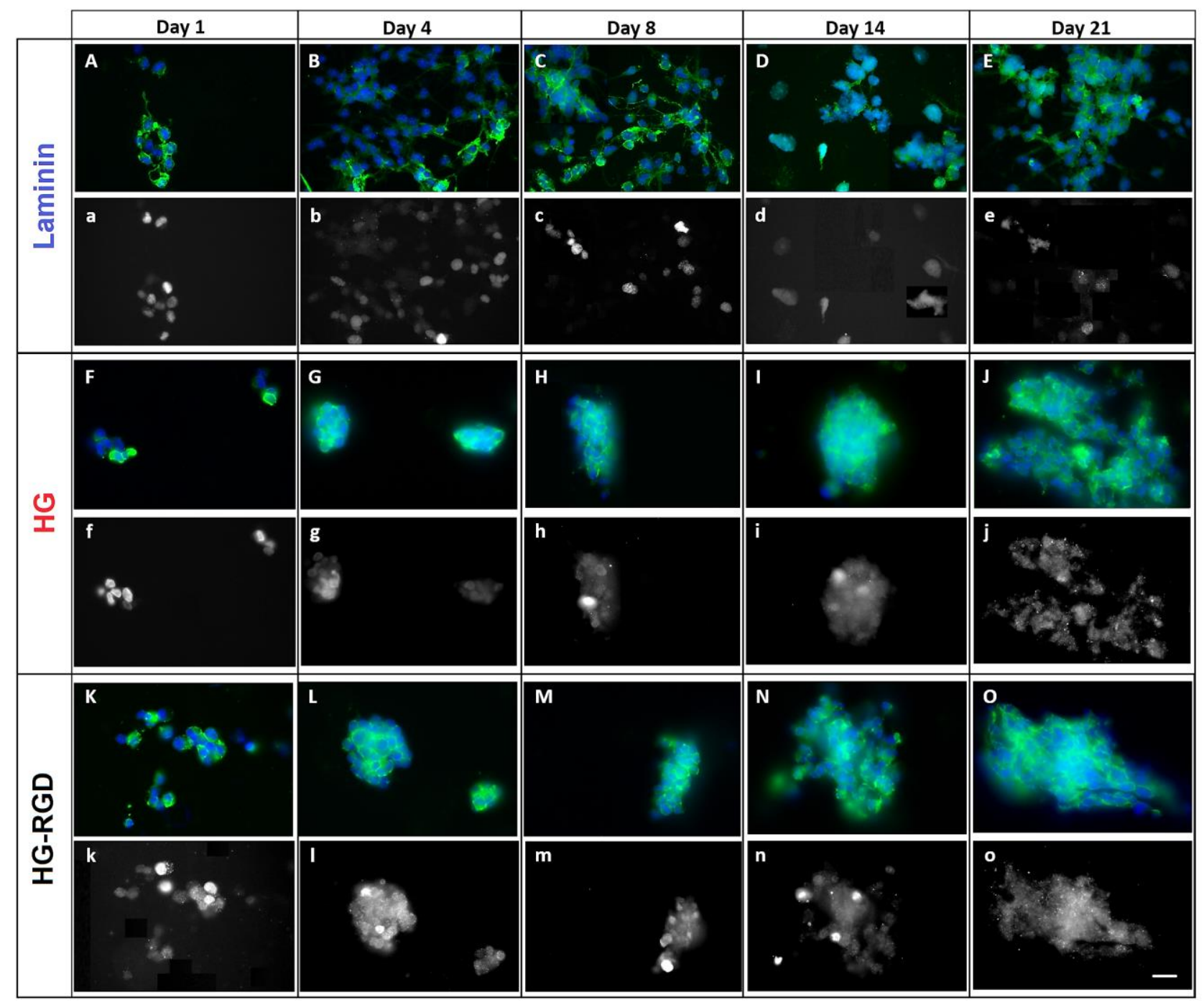

Figure 2. Confocal representative images (63x) of NSCs stained with DAPI and GFP (upper case letters, DAPI in blue and GFP in green) and stained with ki67 (lower case letters, ki67 in white) in laminin (Aa, Bb, $\mathrm{Cc}, \mathrm{Dd}, \mathrm{Ee}), \mathrm{HG}(\mathrm{Ff}, \mathrm{Gg}, \mathrm{Hh}, \mathrm{Ii}, \mathrm{Jj})$ and HG-RGD (Kk, Ll, Mm, Nn, Oo) samples. Images were recorded at different time points $(1,4,8,14$ and 21 days after the cell seeding) and were related to $30-\mu \mathrm{m}$ thick sections for HG and HG-RGD and from glass coverslip in laminin samples. Scale bar represents $30 \mu \mathrm{m}$. 
Seeded cells over the 2D substrate were able to proliferate and rapidly grow achieving a condition close to confluence at day 8 . The high increment of cell population progressively reduced the number of NSCs in non-quiescent state as can be qualitatively observed in the decrease of number of cells ki67 positive (Figures 2a, b, c, d, e). In both hydrogel systems NSCs were able to adhere to the polymer chains and proliferate, giving rise to the neurosphere generation. The fraction of ki67 positive NSCs (cells in active phases cellular cycle) in HG and HG-RGD was reported in Figure 3 and compared to the obtained results with laminin substrate, over time.

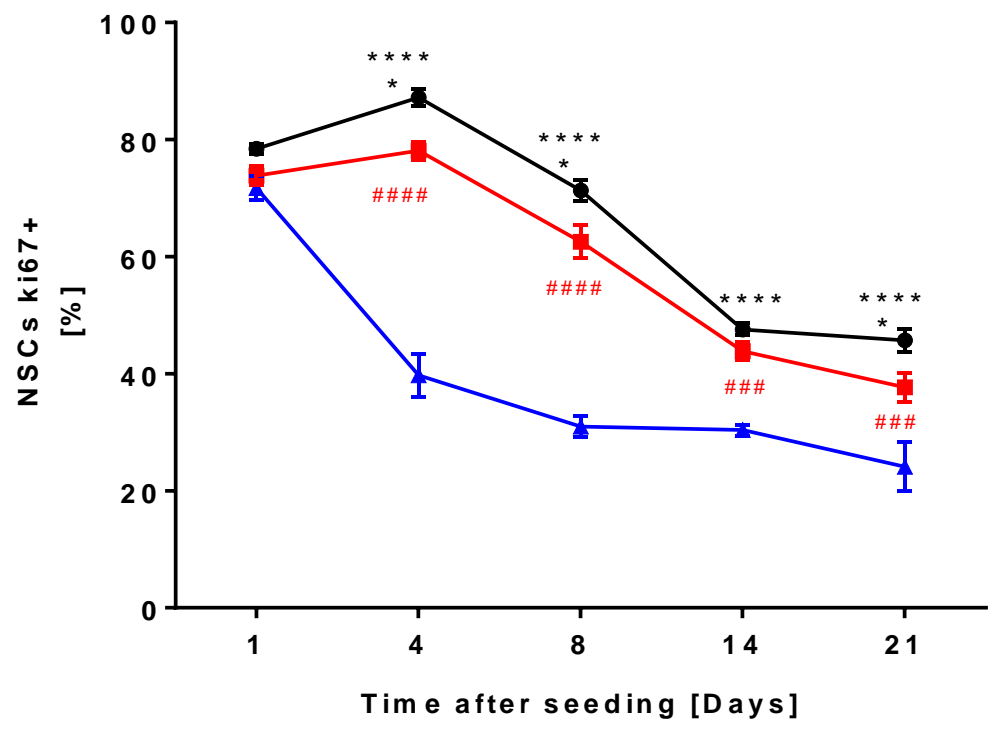

Figure 3. Graph representing the ki67 positive NSCs trends in laminin ( $\Delta$, blue), HG ( $\square$, red) and HG-RGD (O, black) samples, over time. Statistical analysis: two-way ANOVA followed by Tukey's post hoc test. Mean \pm SEM are reported; $\mathrm{n}=6$ per group. $(*) \mathrm{p}<0.05$ vs $\mathrm{HG},(* * * *) \mathrm{p}<0.0001$ vs laminin; $\left(^{(\# \#)} \mathrm{p}<0.001\right.$ vs laminin, ${ }^{\# \# \#)} \mathrm{p}<0.0001$ vs laminin.

Significant different trends can be observed in 3D substrates against laminin. One day after the seeding the percentage of NSCs expressing ki67 was $71 \%$ in 2D system, $74 \%$ in HG sample and slightly above (78\%) in hydrogels with RGD. At day 4, a significant reduction of non-quiescent cells characterized the laminin monolayer (only $43 \%$ of NPCs were stained with the antibody), whereas HG showed that $78 \%$ of cells were in active phases and in HG-RGD up to $87 \%$. 
At day 8, the fraction of NSCs expressing ki67 was further reduced to $31 \%$ in laminin seeding; instead, it was $63 \%$ in $\mathrm{HG}$ and $71 \%$ in HG-RGD.

Monitoring also at 14 and 21 days after the seeding, non-quiescent NSCs decreased in each sample, until $24 \%$ in laminin substrate, $42 \%$ in $\mathrm{HG}$ and $50 \%$ in HG-RGD. Accordingly to these data, the fraction of dead cells in 2D configuration increased over time: NSCs labelled PI achieved the values of $22 \%$ after 8 days and $60 \%$ after 21 days. Instead, in HG and HG-RGD networks, the corresponding percentages were around $2 \%$ until 8 days and only $6 \%$ in $\mathrm{HG}$ and $9 \%$ in RGD modified scaffold at 21 days (ESI Figure S2). These considerations suggest that use of a 3D polymeric tool appears as a promising NSC culture system to give the opportunity to cells to migrate and proliferate for a longer time than 2D culture: we proposed a biocompatible and nontoxic system (ESI, Figure S3) able to provide more superficial area where cells can adhere and grow without spatial restrictions due to the hydrogel mesh size and swelling behavior mimicking the natural environmental niche. Furthermore, focusing on the comparison between ki67 positive NSCs trends in unmodified and RGD-functionalized hydrogels, some differences could be recorded: in particular at day 4 and day 8, HG-RGD sample was characterized by more proliferating cells than HG (Figure 3). The bioactivity of hydrogel was improved by the presence of bioactive molecules such as RGD, which is generally detectable in native ECM. After the seeding, the effect of the tripeptide, in addition to support cell adhesion, is to improve the design of a hospitable 3D environment resembling to NSC natural environment, more than HG system. Another contribution could be the one related to stiffness: from Figure 1D it is visible that the storage modulus of HGRGD is higher than the one of HG. In this direction several studies already proved the role of hydrogel elasticity in 3D cell culture systems ${ }^{52,53}$. The RGD motif influence was well visible at day 4 and day 8, whereas it became restricted over time (in particular at day 14, where the difference of proliferating NSCs between HG and HG-RGD was about 2\%). 
This aspect could be explained considering the continuous increase of cell population in the 3D scaffolds. Performed LDH test (Figure 4) shows the cytotoxicity of the medium surrounding cells in each sample, directly related to the lack of nutrients and all other fundamental constituents of the cell medium.

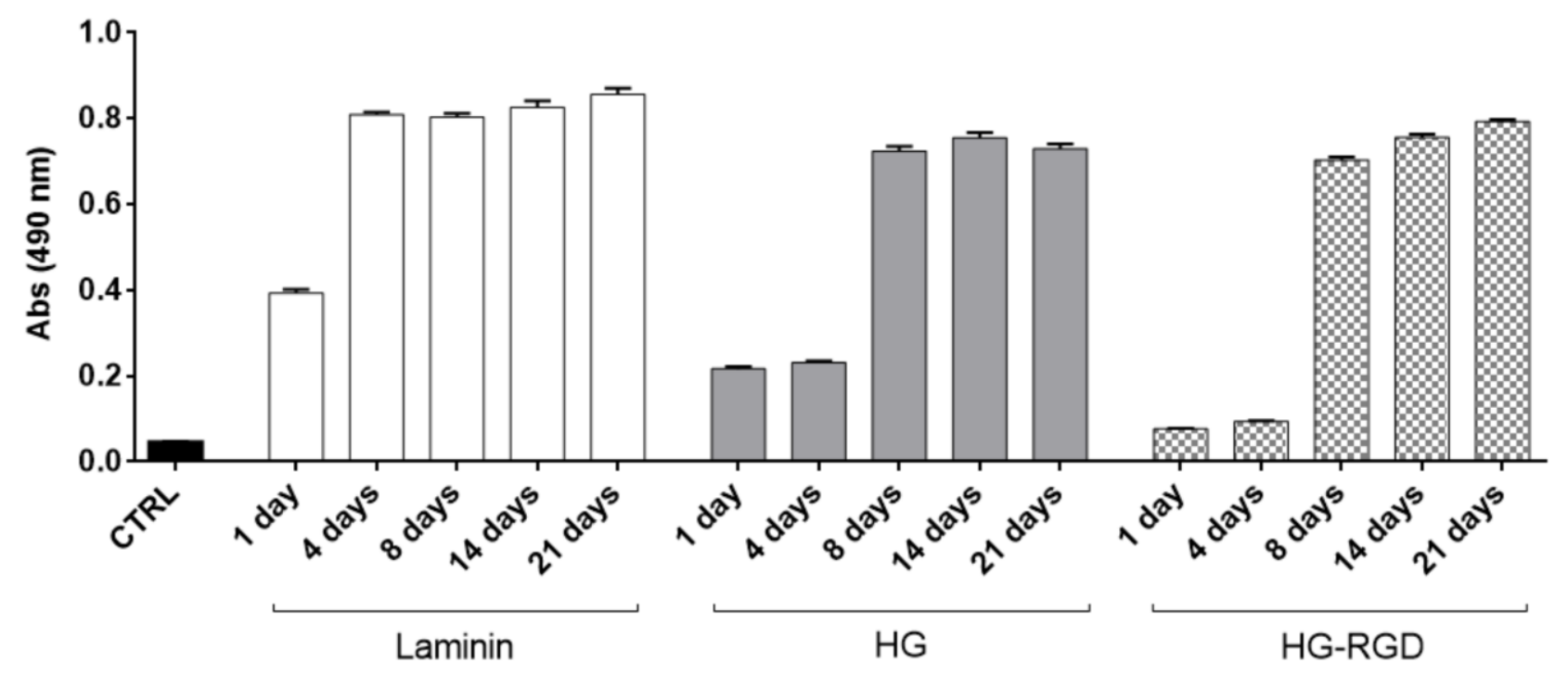

Figure 4. LDH assay for NSCs treated with conditioned media on laminin, HG and HG-RGD culture systems, at different time points. CTRL represents LDH release of NSCs in standard growth medium.

By taking the media conditioned by each sample and using it as cell culture media for new NSCs we observed that lactate dehydrogenase release was prominent for cells treated with the $14^{\text {th }}$ and $21^{\text {st }}$ day HG and HG-RGD media. This suggested that the conditioned media at the indicated time points were poor of nutrients and the latter were completely absorbed by NSCs grafted to the hydrogel network: the high proliferation of cells over time lead to quickly exploit the medium components and gave rise to a condition where RGD benefic effect became limited in the corresponding hydrogel system. 
On the other hand, conditioned media at $1^{\text {st }}$ and $4^{\text {th }}$ day related to the gel scaffolds seemed to be suitable to support cell cultures. In laminin samples, LDH release was significantly high already at 4 days after the NSC seeding. Referring to the different NSC behavior and proliferation trends in 2D and 3D culture systems, LDH release reached maximal values earlier in laminin than in HG and HG-RGD and this trend was maintained over time. Moreover, in HG-RGD sample the recorded absorbance values were lower than in HG at day 1 and day 4. These results confirmed that the design of 3D culture systems allowed improving the cell viability and healthy state respect to a $2 \mathrm{D}$ configuration. 


\section{Conclusions}

In this work, we proposed the synthesis of hydrogels as sustainable $3 \mathrm{D}$ culture systems able to preserve NSC viability and proliferation for longer time than a monolayer configuration. The materials were completely biocompatible and the introduction of RGD motif produced beneficial results on cell healthy state until 8 days after the seeding, allowing a higher number of nonquiescent cells than the corresponding hydrogel without tripeptide modification. In conclusion, RGD-functionalized hydrogels appeared as an optimized scaffold to maintain more suitable biological conditions for neural stem cells and could be consider as a candidate to grow, store and preserve NSCs through a 3D cell culture approach.

\section{Acknowledgments}

Authors would like to thank Dr. Simonetta Papa, Dr. Pietro Veglianese and Stefano Rimondo for fruitful discussion. This work was supported by a core support grant from the Wellcome Trust and Medical Research Council to the Wellcome Trust - MRC Cambridge Stem Cell Institute. LPJ was supported by a research training fellowship from the Wellcome Trust (RRZA/057 RG79423). 


\section{Reference}

1. Y.-H. Tsou, J. Khoneisser, P.-C. Huang and X. Xu, Bioact. Mater., 2016, 1, 39-55.

2. Y. Tabata, J. R. Soc. Interface, 2009, 6, S311-S324.

3. L. Li, Z.-Y. He, X.-W. Wei and Y.-Q. Wei, Regen. Biomater., 2016, 3, 99-105.

4. M. Muskovich and C. J. Bettinger, Adv. Healthc. Mat., 2012, 1, 248-266.

5. T. Zako and M. Maeda, Biomater. Sci., 2014, 2, 951-955.

6. E. Mauri, P. Veglianese, S. Papa, A. Mariani, M. De Paola, R. Rigamonti, G. M. F. Chincarini, I. Vismara, S. Rimondo, A. Sacchetti and F. Rossi, RSC Adv., 2017, 7, 3034530356.

7. E. Mauri, F. Rossi and A. Sacchetti, Mat. Sci. Eng. C, 2016, 61, 851-857.

8. M. Mekhail, G. Almazan and M. Tabrizian, Biomater. Sci., 2015, 3, 279-287.

9. M. Bongio, J. J. J. P. van den Beucken, M. R. Nejadnik, S. C. G. Leeuwenburgh, L. A. Kinard, F. K. Kasper, A. G. Mikos and J. A. Jansen, Eur. Cell. Mater., 2011, 22, 359-376.

10. C. Cha, W. B. Liechty, A. Khademhosseini and N. A. Peppas, ACS Nano, 2012, 6, 93539358.

11. J. C. DiNunzio and R. O. Williams, Drug Dev. Ind. Pharm., 2008, 34, 1141-1167.

12. P. J. Horner and F. H. Gage, Nature, 2000, 407, 963-970.

13. R. C. Assuncao-Silva, E. D. Gomes, N. Sousa, N. A. Silva and A. J. Salgado, Stem Cells Int., 2015, DOI: Artn 948040 10.1155/2015/948040.

14. P. Lu, L. L. Jones, E. Y. Snyder and M. H. Tuszynski, Exp. Neurol., 2003, 181, 115-129.

15. B. Sandner, P. Prang, A. Blesch and N. Weidner, Stem Cells Biol. Reg., 2015, DOI: 10.1007/978-1-4939-1908-6_9, 155-174.

16. G. Martino, S. Pluchino, L. Bonfanti and M. Schwartz, Physiol. Rev., 2011, 91, 1281-1304.

17. C. Cossetti, C. Alfaro-Cervello, M. Donega, G. Tyzack and S. Pluchino, Cell Tissue Res., 2012, 349, 321-329.

18. S. Pluchino, L. Zanotti, M. Deleidi and G. Martino, Brain Res. Rev., 2005, 48, 211-219. 
19. E. Giusto, M. Donega, C. Cossetti and S. Pluchino, Exp. Neurol., 2014, 260, 19-32.

20. I. Elliott Donaghue, C. H. Tator and M. S. Shoichet, Biomater. Sci., 2015, 3, 65-72.

21. R. Edmondson, J. J. Broglie, A. F. Adcock and L. J. Yang, Assay Drug Dev. Technol., 2014, 12, 207-218.

22. S. Knowlton, Y. K. Cho, X. J. Li, A. Khademhosseini and S. Tasoglu, Biomater. Sci., 2016, 4, 768-784.

23. F. Rossi, G. Perale, G. Storti and M. Masi, J. Appl. Polym. Sci., 2012, 123, 2211-2221.

24. L. Beria, T. N. Gevrek, A. Erdog, R. Sanyal, D. Pasini and A. Sanyal, Biomater. Sci., 2014, 2, 67-75.

25. F. Ullah, M. B. H. Othman, F. Javed, Z. Ahmad and H. M. Akil, Mat. Sci. Eng. C, 2015, 57, 414-433.

26. Y. S. Zhang and A. Khademhosseini, Science, 2017, 356, eaaf3627.

27. X. W. Li, E. Katsanevakis, X. Y. Liu, N. Zhang and X. J. Wen, Progr. Polym. Sci., 2012, 37, 1105-1129.

28. I. Caron, F. Rossi, S. Papa, R. Aloe, M. Sculco, E. Mauri, A. Sacchetti, E. Erba, N. Panini, V. Parazzi, M. Barilani, G. Forloni, G. Perale, L. Lazzari and P. Veglianese, Biomaterials, 2016, 75, 135-147.

29. R. R. Yang, C. X. Xu, T. Wang, Y. Q. Wang, J. N. Wang, D. P. Quan and D. Y. B. Deng, RSC Adv., 2017, 7, 41098-41104.

30. P. Naghdi, T. Tiraihi, F. Ganji, S. Darabi, T. Taheri and H. Kazemi, J. Tissue Eng. Regen. Med., 2016, 10, 199-208.

31. A. Sacchetti, E. Mauri, M. Sani, M. Masi and F. Rossi, Tetrahedron Lett., 2014, 55, 68176820.

32. F. Rossi, R. Ferrari, S. Papa, D. Moscatelli, T. Casalini, G. Forloni, G. Perale and P. Veglianese, Colloid Surf. B, 2013, 108, 169-177. 
33. S. Pluchino, A. Quattrini, E. Brambilla, A. Gritti, G. Salani, G. Dina, R. Galli, U. Del Carro, S. Amadio, A. Bergami, R. Furlan, G. Comi, A. L. Vescovi and G. Martino, Nature, 2003, 422, 688-694.

34. M. Cusimano, D. Biziato, E. Brambilla, M. Donega, C. Alfaro-Cervello, S. Snider, G. Salani, F. Pucci, G. Comi, J. M. Garcia-Verdugo, M. De Palma, G. Martino and S. Pluchino, Brain, 2012, 135, 447-460.

35. A. Follenzi, L. E. Ailles, S. Bakovic, M. Geuna and L. Naldini, Nat. Genet., 2000, 25, 217222.

36. U. Hersel, C. Dahmen and H. Kessler, Biomaterials, 2003, 24, 4385-4415.

37. A. Lagunas, J. Comelles, E. Martínez, E. Prats-Alfonso, G. A. Acosta, F. Albericio and J. Samitier, Nanomedicine, 8, 432-439.

38. A. C. de Luca, A. Faroni, S. Downes and G. Terenghi, J. Tissue Eng. Regen. Med., 2016, 10, 647-655.

39. M. Taddei, S. Ferrini, L. Giannotti, M. Corsi, F. Manetti, G. Giannini, L. Vesci, F. M. Milazzo, D. Alloatti, M. B. Guglielmi, M. Castorina, M. L. Cervoni, M. Barbarino, R. Fodera, V. Carollo, C. Pisano, S. Armaroli and W. Cabri, J. Med. Chem., 2014, 57, 22582274.

40. N. Ma, Y. Wang, B. X. Zhao, W. C. Ye and S. Jiang, Drug Des. Devel. Ther., 2015, 9, 1585-1599.

41. H. B. Li, Q. L. Zheng and C. P. Han, Analyst, 2010, 135, 1360-1364.

42. C. O. Kappe, Angew. Chem. Int. Ed., 2004, 43, 6250-6284.

43. E. Varoni, M. Tschon, B. Palazzo, P. Nitti, L. Martini and L. Rimondini, Connect. Tissue Res., 2012, 53, 548-554.

44. M. Xu, J. Q. Zhu, F. F. Wang, Y. J. Xiong, Y. K. Wu, Q. Q. Wang, J. Weng, Z. H. Zhang, W. Chen and S. J. Liu, ACS Nano, 2016, 10, 3267-3281. 
45. T. Gros, J. S. Sakamoto, A. Blesch, L. A. Havton and M. H. Tuszynski, Biomaterials, 2010, 31, 6719-6729.

46. J. M. Zhu, C. Tang, K. Kottke-Marchant and R. E. Marchant, Bioconjug. Chem., 2009, 20, 333-339.

47. A. P. Pego, S. Kubinova, D. Cizkova, I. Vanicky, F. M. Mar, M. M. Sousa and E. Sykova, J. Cell. Mol. Med., 2012, 16, 2564-2582.

48. G. Perale, F. Rossi, M. Santoro, M. Peviani, S. Papa, D. Llupi, P. Torriani, E. Micotti, S. Previdi, L. Cervo, E. Sundstrom, A. R. Boccaccini, M. Masi, G. Forloni and P. Veglianese, J. Control. Release, 2012, 159, 271-280.

49. M. Santoro, P. Marchetti, F. Rossi, G. Perale, F. Castiglione, A. Mele and M. Masi, J. Phys. Chem. B, 2011, 115, 2503-2510.

50. E. Knight and S. Przyborski, J. Anat., 2015, 227, 746-756.

51. J. Vanderburgh, J. A. Sterling and S. A. Guelcher, Ann. Biomed. Eng., 2017, 45, 164-179.

52. A. J. Engler, S. Sen, H. L. Sweeney and D. E. Discher, Cell, 2006, 126, 677-689.

53. W. J. Hadden, J. L. Young, A. W. Holle, M. L. McFetridge, D. Y. Kim, P. Wijesinghe, H. Taylor-Weiner, J. H. Wen, A. R. Lee, K. Bieback, B.-N. Vo, D. D. Sampson, B. F. Kennedy, J. P. Spatz, A. J. Engler and Y. S. Choi, Proc. Natl. Acad. Sci. USA, 2017, 114, 5647-5652. 